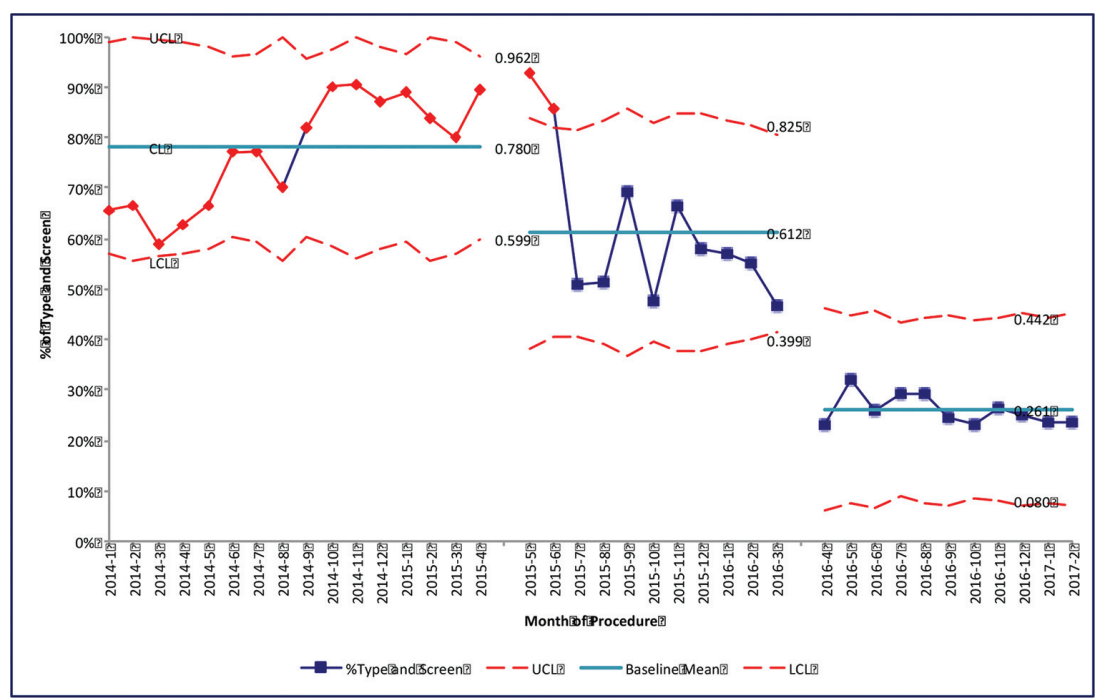

Abstract 1050 Figure 1 Percentage of type and screen drawn for laparoscopic hysterectomy procedure $p$ chart, sigma

(21\%), 5 patients (1\%) received transfusion (Table 1). Figure-1 shows a process control chart plotting the $\mathrm{T}$ and $\mathrm{S}$ samples over time with interventions at 5-2015 and 4-2016 which show a significant decrease in mean $\mathrm{T}$ and $\mathrm{S}$ samples.

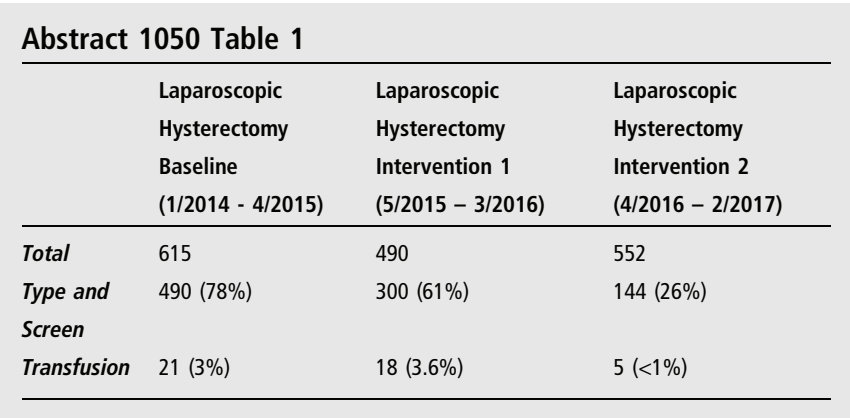

Conclusions Unnecessary preparation of blood products for operations with historically low rates of transfusion represents wasted phlebotomy, labour, and expense. Using laparoscopic hysterectomy as an example, we decreased unnecessary $\mathrm{T}$ and $\mathrm{S}$ using data to guide pre-operative testing.

\section{REDUCING UNWANTED AND UNWARRANTED ED AND HOSPITAL UTILISATION FOR FRAIL ELDERS IN RURAL SKILLED NURSING FACILITIES: A HYBRID IMPROVEMENT-IMPLEMENTATION APPROACH}

${ }^{1}$ Brant Oliver, ${ }^{2}$ Daniel Stadler, ${ }^{2}$ Ellen Flaherty, ${ }^{1}$ Stephen Bartels. 'Dartmouth Collaboratory for Health Care Delivery Science, US; ${ }^{2}$ Dartmouth Hitchcock, US

\subsection{6/bmjoq-2017-IHI.29}

Background Frail elders in residential skilled nursing facilities (SNFs) have high rates of emergency department (ED) utilisation and hospitalizations. We sought to implement and iteratively specify an intervention to improve utilisation and cost outcomes for frail elders in rural SNFs.

Objectives (1) To reduce unwanted and avoidable ED utilisation and hospitalizations; (2) to reduce related costs.

Methods Adopting evidence based practices, we iteratively developed an implementation approach including the following key elements: (1) advanced directives; (2) a dedicated closed- call team of providers following SNF residents; (3) a biweekly case review of all ED referrals and hospitalizations; and (4) a standardised triage communication process. We conducted three PDSA cycles over a 6 month period and assessed clinical and cost outcomes using inferential statistics and statistical process control (SPC) methods.

Results Three rural SNFs participated in the intervention from January-June 2016. Three PDSA cycles were conducted. Monthly hospital-based care utilisation for long term care (LTC) residents reduced from 10 to 3.3 episodes $(\mathrm{p}<0.05)$. ED transfers reduced by $59 \%(\mathrm{p}<0.05)$, and hospitalizations reduced by $62 \% \quad(\mathrm{p}<0.05)$, without associated changes in

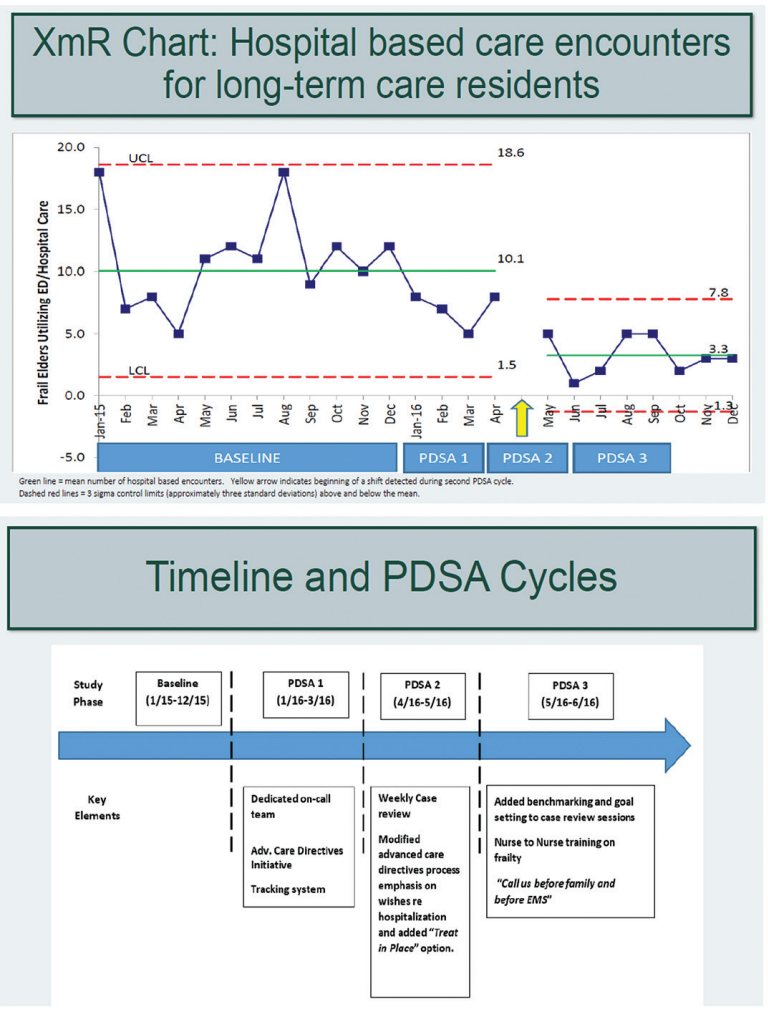

Abstract 1057 Figure 1 
Annual utilization by category:

Pre- (2015) versus post- (2016) intervention
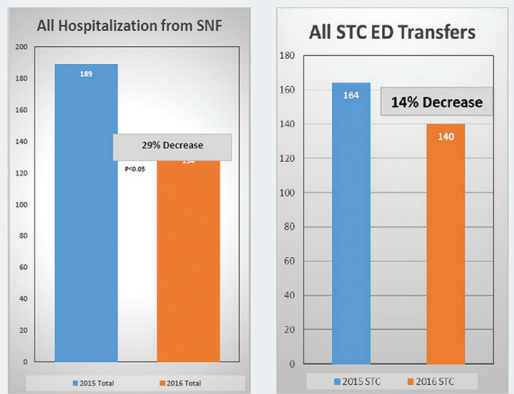

All LTC ED Transfers

Annual utilization by category:

Pre- (2015) versus post- (2016) intervention

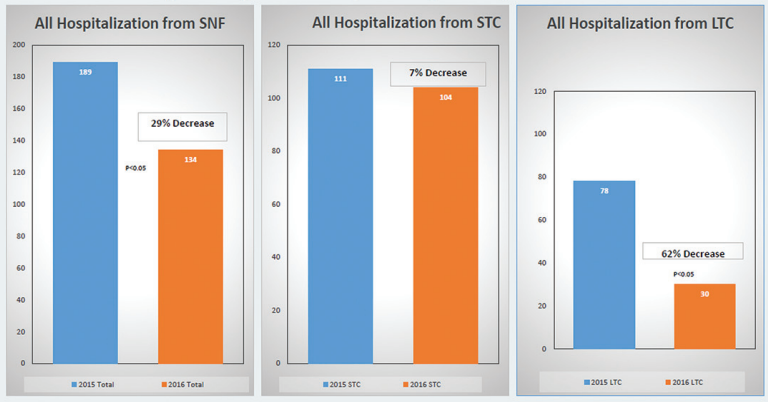

SNF= Skilled Nursing Facility; STC= Short-Term Care residents; LTC= Long-Term Care residents; ED= Emergency Department

\section{Abstract 1057 Figure 2}

overall mortality or quality performance indicators. SPC analysis suggests that PDSA Cycle 2 was temporally related to the onset of improvement. Estimated cost savings calculated based on utilisation reductions is approximately $\$ 7$ million annually.

Conclusions This initial pilot was feasible, achieved significant outcomes, and is an example of the use of improvement methods to iteratively develop and optimise an implementation approach. This approach has potential to significantly impact outcomes, utilisation and cost and is worthy of continued study.

Cost Considerations:

Pre- (2015) versus post- (2016) intervention findings and estimated savings

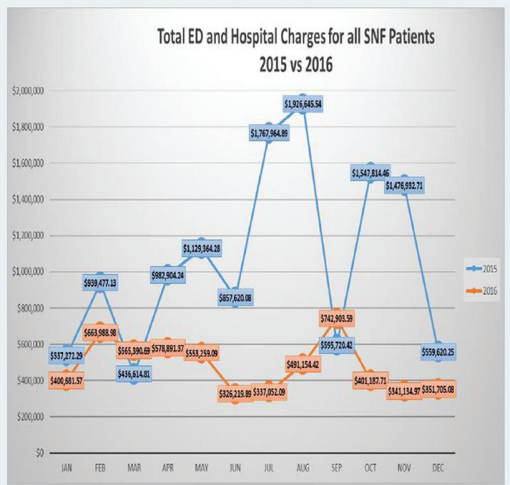

Total ED and Hospitalization

charges for all SNF

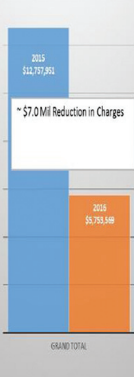

Abstract 1057 Figure 3

\section{REDUCING EMERGENCY DEPARTMENT UTILISATION IN INFANTS: A QUALITY IMPROVEMENT STUDY}

${ }^{1} J e n n y$ Chan, ${ }^{2}$ Mariam Maloyan, ${ }^{2}$ Hannah Durant, ${ }^{2}$ Ellen Reisinger, ${ }^{2}$ Pamela Schubert, ${ }^{2}$ Grace Chi, ${ }^{2}$ Joanne Cox, ${ }^{2}$ Kathleen Conroy. 'Boston Children's Hospital Primary Care at Longwood, US; ${ }^{2}$ Boston Children's Hospital Primary Care at Martha Eliot, US

\subsection{6/bmjoq-2017-|HI.30}

Background Utilising the Emergency Department (ED) rather than the primary care medical home (PCMH) for non-emergent care results in decreased continuity and increased medical expense. Young, low-income children have the highest lowacuity ED visit rates. Few PCMH-based interventions have been shown to decrease unnecessary ED use.

Objectives Reduce rates of low-acuity ED utilisation in children under 12 months.

Methods This was a quality improvement study set in an academic primary care clinic serving 15,000 predominantly lowincome families. Interventions focused on expanding urgent care (UC) and nursing-line access, improving parents' awareness of these ED-alternatives, and enhancing caregiver knowledge of infant care (Figure 1). Our primary outcome was the 12 month rolling rate of low-acuity ED visits; high-acuity ED visits acted as a balancing measure.

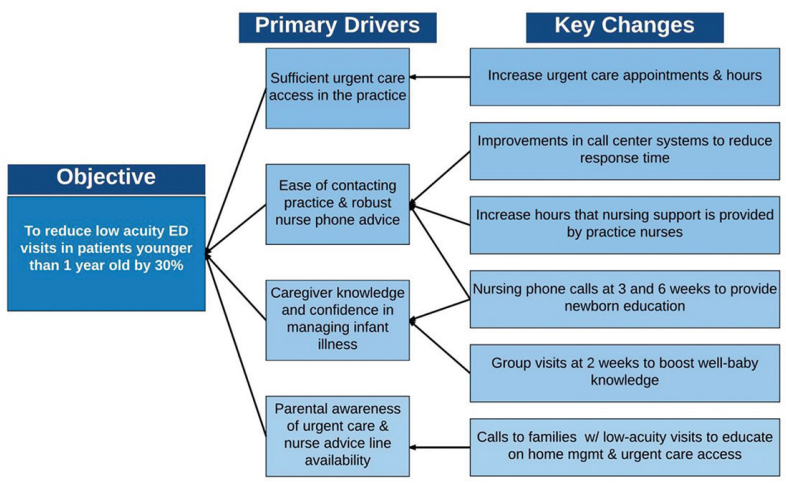

Abstract 1058 Figure 1 Driver diagram

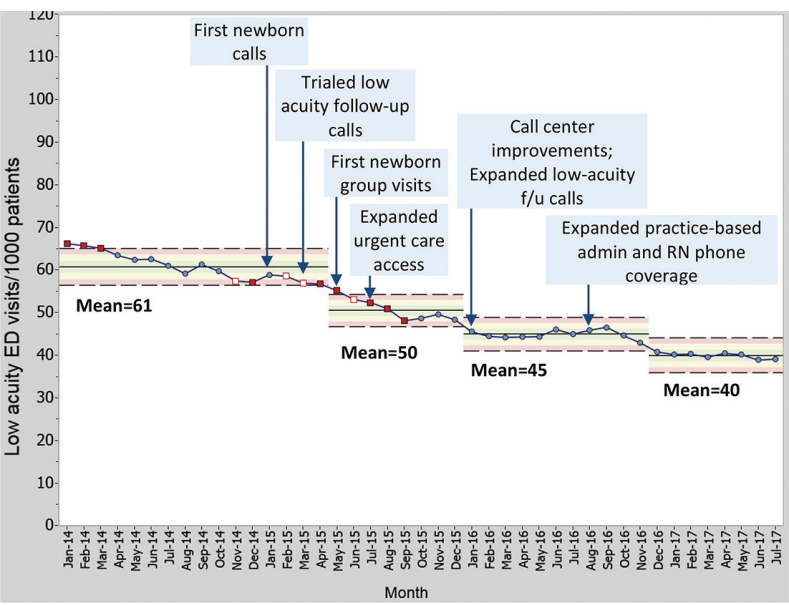

Abstract 1058 Figure 2 ED utilisation by patients $<1$ year, 12 month rolling rate (low acuity visits) 